\title{
Characterization of Serum MicroRNAs Profile of PCOS and Identification of Novel Non-Invasive Biomarkers
}

\author{
Wei Long a,e Chun Zhao be Chenbo Jic Hongjuan Dinga Yugui Cuid Xirong Guo ${ }^{c}$ \\ Rong Shen ${ }^{\mathrm{a}}$ Jiayin Liu ${ }^{\mathrm{d}}$
}

a'Department of Obstetrics and Gynecology, Nanjing Maternity and Child Health Care Hospital, Nanjing Medical University, ${ }^{b}$ Department of Reproductive Medicine, Nanjing Maternity and Child Health Care Hospital, Nanjing Medical University, 'Department of Children Health Care, Nanjing Maternity and Child Health Care Hospital, Nanjing Medical University, 'State Key Laboratory of Reproductive Medicine, Clinical Center of Reproductive Medicine, First Affiliated Hospital, Nanjing Medical University, Nanjing



\section{Key Words \\ PCOS • MicroRNA • Microarray • Biomarkers}

\begin{abstract}
Background: Polycystic ovary syndrome (PCOS), the most common endocrinopathy in women of reproductive age, is characterized by polycystic ovaries, chronic anovulation, hyperandrogenism and insulin resistance. Despite the high prevalence of hyperandrogenemia, a definitive endocrine marker for PCOS has so far not been identified. Circulating miRNAs have recently been shown to serve as diagnostic/prognostic biomarkers in patients with cancers. Our current study focused on the altered expression of serum miRNAs and their correlation with PCOS. Method and Results: We systematically used the TaqMan Low Density Array followed by individual quantitative reverse transcription polymerase chain reaction assays to identify and validate the expression of serum miRNAs of PCOS patients. The expression levels of three miRNAs (miR-222, miR-146a and miR-30c) were significantly increased in PCOS patients with respect to the controls in our discovery evaluation and followed validation. The area under the receiver operating characteristic (ROC) curve (AUC) is $0.799,0.706$, and 0.688 , respectively. The combination of the three miRNAs using multiple logistic regression analysis showed a larger AUC (0.852) that was more efficient for the diagnosis of PCOS. In addition, logistic binary regression analyses show miR-222 is positively associated with serum insulin, while miR-146a is negatively associated with serum testosterone. Furthermore, bioinformatics analysis indicated that the predicted targets function of the three miRNAs mainly involved in the metastasis, cell cycle, apoptosis and endocrine. Conclusion: Serum miRNAs are differentially expressed between PCOS patients and controls. We identified and validated a class of three serum miRNAs that could act as novel non-invasive biomarkers for diagnosis of PCOS. These miRNAs may be involved in the pathogenesis of PCOS.
\end{abstract}

Copyright $(2014$ S. Karger AG, Basel

Jiayin Liu

and Rong Shen
State Key Laboratory of Reproductive Medicine, Clinical Center of Reproductive Medicine, First Affiliated Hospital, Nanjing Medical University, Nanjing 210029 (China); and Department of Obstetrics and Gynecology, Nanjing Maternity and Child Health Care Hospital, Nanjing Medical University, Nanjing 210009, (People's Republic of China) E-Mail Jyliu_nj@126.com and E-Mail Shenr_njfy@126.com 


\section{Introduction}

Polycystic ovary syndrome (PCOS), a multifactorial endocrine condition, affects up to $5-10 \%$ of women in reproductive age and accounts for $75 \%$ of anovulatory infertility [1-3]. PCOS is a heterogeneous syndrome with complex pathologies with follicle growth arrest at the small antral stage, minimal granulosa cell proliferation, hyperthecosis and hyperandrogenemia, and chronic anovulation [1]. It is associated not only with infertility, but also with increased risk of metabolic disorders, such as insulin resistance, diabetes, obesity, and cardiovascular diseases [4]. Both genetic and environmental factors are known to contribute to the pathogenesis of PCOS; Genomic studies in PCOS have revealed a variety of altered genes that fall into many functional categories, including cell division, apoptosis and regulation of gene expression and metabolism [5]. However, to date, the etiology of PCOS is not fully understood.

MicroRNAs (miRNAs) are single-stranded and small noncoding RNA molecules of 18-24 nt in length that negatively regulate the expression of target genes in a posttranscriptional manner, by binding to messenger RNAs in a sequence-specific manner [6]. These short molecules are conserved from worms to mammals; this high conservation of miRNAs sequences highlights the significance of their function. They contribute to the regulation of a variety of biological functions across diverse organisms such as apoptosis, proliferation, differentiation, development, cell cycle, stem cell maintenance, metabolism and hematopoiesis [7-9].

Recently, the role of microRNAs (miRNAs) has been studied in the pathogenesis of endometriosis and associated reproductive conditions [10]. Differences in miRNA profiles in from women with PCOS compared with those from donor fertile controls have been shown [11]. However, the serum miRNA profile of PCOS has not been reported. miRNAs were found to be abundant in human serum, and the serum miRNAs attracted most attention because of their unique merits (i.e., stable, easily to be detected, and potentially disease-specific) [12]. Although the exact mechanism how miRNAs enter into the serum and whether they are biologically functional or simply biomarkers for unknown etiological factors is still a big obsession, a recent study reported that miRNAs could be selectively packaged into microvesicles and actively secreted [13]. Therefore, serum miRNAs could be a potential independent predictive system for different diseases, compared with biomarkers derived from target tissues.

Herein, we hypothesized that serum miRNA could serve as candidate biomarkers for the diagnosis of PCOS. To address this hypothesis, we systematically screened serum miRNAs by using the Taqman Low Density Array (TLDA) chips followed by individual quantitative reverse transcriptase polymerase chain reaction (RT-qPCR) assays systematically and then performed RT-qPCR assays to validate them in the large sample.

\section{Materials and Methods}

\section{Patients and Study design}

We designed a multistage retrospective nested case-control study to determine whether serum miRNA profiling could predict PCOS development and whether the predicting property may be prior to the change of serum insulin and androgen. From February 2011 to October 2013, 68 patients diagnosed with PCOS in the Nanjing Maternity and Child Health Hospital were enrolled in the PCOS group (according to PCOS diagnostic criteria revised in the 2003 Rotterdam meeting). The age of patients ranged from 23.8 to 29.4 years, who had no history of drugs affecting glucose and lipid metabolism. Age-matched healthy controls (n =68) were recruited from a mandatory screening program for employees instituted at the Nanjing Maternity and Child Health Hospital. Exclusion criteria in controls included any known medical conditions or diseases. Care was taken to exclude hyperandrogenism and chronic anovulation. The age of the controls ranged from 24.5 to 31.3 years. This study was conducted in accordance with the declaration of Helsinki. This study was 
conducted with approval from the Ethics Committee of the Nanjing Maternity and Child Health Hospital of Nanjing Medical University. Written informed consent was obtained from all participants.

The study was divided into two stepwise phases. In phase I (biomarker discovery), we randomly pooled the serum of five women with PCOS and five matched controls, respectively, to identify the differential miRNAs profile by TLDA chip assays between these two groups. By comparing the relative expression level of serum miRNAs, significantly up- or down-regulated miRNAs were preliminarily selected for further analysis in the next phase. In the phase II, we subsequently conducted quantitative reverse transcription-polymerase chain reaction (RT-qPCR) on 68 women with PCOS and 68 Normal controls to validate the miRNAs which was initial screened. Depending on the results of this phase, receiver operating characteristic (ROC) curvebased risk assessment analysis was conducted to assess the sensitivity and specificity of maternal serum for predicting PCOS.

\section{Serum preparation and RNA extraction}

Five-ml venous blood was collected from each participant using a procoagulant drying tube. The whole blood was separated into serum and cellular fractions by centrifugation at 4,000 rpm for $10 \mathrm{~min}$, followed by $12000 \mathrm{rpm}$ for $15 \mathrm{~min}$ to completely remove cell debris. Isolation of serum total RNA was described previously with some modifications [14]. In brief, the Trizol Reagent (Invitrogen, Carlsbad, CA) was used for serum denaturizing and Qiagen miRNeasy Mini kit (Qiagen, Valencia, CA) for RNA collection and purification according to the manufacturer's protocol. Because there was no consensus on the use of housekeeping miRNA for the serum RT-qPCR analysis, after the initial denaturizing step, we routinely spiked in synthetic C.elegans miR-39 to a final concentration of $1024 \mathrm{pmol} / \mathrm{ml}$ for all samples in order to control variations in RNA extraction and/or purification procedures because of the absence of homologous sequences in humans [15]. Furthermore, all study subjects were recruited during the same period, stored under the same conditions (for each center), and the samples were handled in equal volume in each experiment step to control the potential bias.

\section{TLDA chip assays and RT-qPCR}

In the discovery stage, we used TLDA Chips (human microRNA panel V2.0, Applied Biosystems Inc, CA, USA) to screen differentially expressed miRNAs from the two pooled samples. A total of $960-\mathrm{ml}$ serum from each pool (5 samples) was used. Megaplex RT reactions and pre-amplification reactions were run according to the manufacture's protocol, in which $75 \mathrm{ml} 0.16 \mathrm{TE}$ was added to PreAmp product, and 9ml diluted PreAmp product was used to run the RT-qPCR reactions by dispensing $100 \mathrm{ml}$ of the PCR reaction mix into each port of the TaqMan MicroRNA Array. The default PCR procedure was used, and the analysis was performed by using RQ manager software (Applied Biosystems Inc.). $\Delta \mathrm{Cq}$ and $\Delta \Delta \mathrm{Cq}$ were calculated using the following mathematical formula: $\Delta \mathrm{CT}=\mathrm{Cq}$ sample-Cq RNU6B $\Delta \Delta \mathrm{Cq}=\Delta \mathrm{Cq}$ case $-\Delta \mathrm{Cq}$ control. Finally, the $\Delta \Delta \mathrm{Cq}$ was normalized against the cel-miR-39. Then, we used TaqMan microRNA probes (Applied Biosystems Inc.) to perform RT-qPCR assays according to the manufacturer's instructions [16]. The probe information was shown in Table 1. Equal volume of samples was used in each step from serum purification to RT-qPCR. The total RNA was reverse-transcribed to cDNA by using a TaqMan microRNA RT Kit and stem-loop RT primers (Applied Biosystems Inc.). RT-qPCR was performed using the TaqMan PCR kit on the ABI 7900 Real-Time PCR System (Applied Biosystems Inc.). The reactions were initiated in a 384-well optical plate at $95^{\circ} \mathrm{C}$ for $5 \mathrm{~min}$, followed by 40 cycles of $95^{\circ} \mathrm{C}$ for $15 \mathrm{~s}$ and $60^{\circ} \mathrm{C}$ for $1 \mathrm{~min}$. We assigned equal number of patients and controls on one plate and run the RT-PCR for target miRNAs and cel-miR-39 simultaneously. All reactions, including no-template controls, were run in triplicate. The Cq values were determined using the fixed threshold settings. The relative expression levels of target miRNAs were determined by the equation $2^{-\Delta \mathrm{Cq}}$, in which $\Delta \mathrm{Cq}=\mathrm{Cq}$ sample $-\mathrm{C}$ q cel-39

\section{Reverse transcription (RT) and quantitative PCR ( $q P C R$ )}

The total RNA was reverse-transcribed to cDNA with the TaqMan MicroRNA reverse transcription kit and miRNA-specific stem-loop primers (Applied Biosystems 9 Inc.). The 15 $\mu$ l reaction mix consisted of $0.15 \mu \mathrm{l}$ of $100 \mathrm{mM}$ dNTP mix, $1 \mu \mathrm{l}$ of Multiscribe RT enzyme ( $5 \mathrm{U} / \mu \mathrm{l}), 1.5 \mu \mathrm{l}$ of $10 \times \mathrm{RT}$ Buffer, $0.19 \mu \mathrm{l}$ of RNase Inhibitor $(20 \mathrm{U} / \mu \mathrm{l}), 7.16 \mu \mathrm{l}$ of nuclease-free water, $3 \mu \mathrm{l}$ of TaqMan RT primer and $2 \mu \mathrm{l}$ total RNA. Reverse transcription was initiated at $16^{\circ} \mathrm{C}$ for $30 \mathrm{~min}, 42^{\circ} \mathrm{C}$ for $30 \mathrm{~min}, 85^{\circ} \mathrm{C}$ for $5 \mathrm{~min}$, and held at $4^{\circ} \mathrm{C}$. Subsequently, real-time PCR was performed intriplicate for each maternal serum sample and no-template negative controls 
included. For a final volume of $20 \mu \mathrm{l}$ reaction, $1 \mu \mathrm{l}$ synthesized cDNA was mixed with $8 \mu \mathrm{l}$ diethylpyrocarbonate (DEPC)-treated water, 10 $\mu$ l TaqMan Gene Expression Master Mix and 1 $\mu$ l TaqMan MicroRNA Assay (Applied Biosystems). The MicroRNA Assay IDs was shown in Table 1. The mixture was incubated at $50^{\circ} \mathrm{C}$ for $2 \mathrm{~min}$, $95^{\circ} \mathrm{C}$ for $10 \mathrm{~min}$, followed by 40 cycles of $95^{\circ} \mathrm{C}$ for $15 \mathrm{~s}$ and $60^{\circ} \mathrm{C}$ for $1 \mathrm{~min}$. Amplification was run using the 96-well on the ABI 7500 Real-Time PCR system (Applied Biosystems) following the manufacturer's instructions. We performed RT-qPCR for the target miRNAs and cel-miR-39 simultaneously to normalize the serum miRNA levels. The relative expression of target miRNA was determined with the comparative cycle

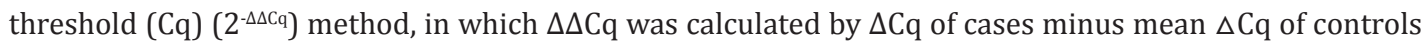
and $\Delta \mathrm{Cq}=$ Cq sample-Cq cel-miR-39.

\section{Bioinformatic analyses for mRNA target and molecular pathway prediction}

GO analysis was applied to analyze the main function of the target genes of miRNAs according to the GO database which provides the key functional classifications for the National Center for Biotechnology Information (NCBI) [17]. Generally, Fisher's exact test and v2 test were used to classify the GO category, and the false discovery rate (FDR) was calculated to correct the $\mathrm{P}$ value.

In order to identify molecular pathways potentially altered by the expression of multiple miRNAs, we used the DIANA-mirPath web-based computational tool [18]. The software performs an enrichment analysis of multiple miRNA target genes comparing each set of miRNA targets to all known KEGG ( Kyoto Encyclopedia of Genes and Genomes ) pathways [19]. The combinatorial effect of co-expressed miRNAs in the modulation of a given pathway is taken into account by the simultaneous analysis of multiple miRNAs. The graphical output of the program provides an overview of the parts of the pathway modulated by miRNAs, facilitating the interpretation and presentation of the analysis results.

\section{Statistical analysis}

All statistical analyses were performed using SPSS software version 17.0 (SPSS, Inc, Chicago, USA). Differences in the demographic and clinical characteristics were estimated by the student's t-test between different groups. The Mann-Whitney U test was used to compare the relative expression level of the maternal serum miRNAs between the PCOS group and controls. For each miRNA, a ROC curve was 68 plotted to evaluate its discriminating effects. The sensitivity and specificity of detecting cases and controls were assessed by the area under the ROC curve (AUC) and 95\% confidence interval (CI). To improve the diagnostic rates of PCOS, we combined the expression levels of the four miRNAs using multiple logistic regression analysis to perform the above risk assessment. All P-values less than 0.05 were considered statistically significant, and all the statistical tests were two-sided.

\section{Results}

\section{Characteristics of participants}

The characteristics of participants are summarized in Table 1. The cases and controls were well matched on age. Body mass index (BMI), insulin(INS), glucose(Glu), estradiol (E2), testosterone(T), PRL and LH were significantly higher in women with PCOS than in controls, whereas FSH were significantly lower in the cases than in the controls.

\section{Phase I: Biomarker-discovery}

The purpose of this study was to screen maternal serum miRNAs as biomarkers for PCOS. We used TaqMan Low Density Array to identify differentially expressed miRNAs in serum samples from five women with PCOS and their corresponding controls who were selected randomly. We calculated the mean expression level and employed fold changes of included miRNAs. On the basis of both scientific and applicable consideration, miRNAs that were $>2$-fold up-regulated or $<0.5$-fold down-regulated changes in the PCOS group were selected as the candidates for the next stage of validation. Following preliminary screening, we found eight up-regulated miRNAs (miR-222, miR-16, miR-19a, miR-106b, miR-30c, miR146a, miR-24 and miR-186) and one down-regulated miRNA (miR-320). Then, the eventual validation of the 9 miRNAs was performed in the phase II. 
Table 1. Primers used in this study

\begin{tabular}{ccc}
\hline Gene name & Forward primer(5'-3') & Reverse primer(5'-3') \\
\hline mir-222 & TGGATCTCCAGCACCTAAGAA & TGTGTGTAATTCAAGGTAAAGTTTTCA \\
mir-16 & GCAGCACGTAAATATTGGCGT & CAGCAGCACAGTTAAATACTGGAG \\
mir-19a & GCATCTACTGCCCTAAGTGCTC & TGCTACAGAAGCTGTCACATCA \\
mir-106b & TAAAGTGCTGACAGTGCAGAT & CAAGTACCCACAGTGCGGT \\
mir-30c & ACCTCAGAAACAAACACGGG & TGGTACTGGGTTCCTGAGG \\
mir-146a & CCATGTTGCCCAGGCTAGT & TCTTCCAAGCTCTTCAGCAG \\
mir-24 & CTCCCGTGCCTACTGAGCT & CCCTGTTCCTGCTGAACTGAG \\
mir-186 & CACCCATCATATTCTTCCCA & GACATTCACATGCTTCAGGTAG \\
mir-320 & CGCCTTCTCTTCCCGGT & TTCGCCCTCTCAACCCA \\
\hline
\end{tabular}

Table 2. Anthropometry and sex steroids measured with mass spectrometry in PCOS women and controls. Values are expressed as mean \pm SD or median (25th-75th interquartile ranges). * P value was obtained using fishing Fishing exact probability method. $\mathrm{P}<0.05$ is considered statistically significant

\begin{tabular}{lccl}
\hline Variable & Control $(\mathrm{n}=68)$ & PCOS $(\mathrm{n}=68)$ & P value \\
\hline AGE $(\mathrm{yr})$ & $27.9 \pm 3.4$ & $26.6 \pm 2.8$ & NS \\
BMI $\left(\mathrm{kg} / \mathrm{m}^{2}\right)$ & $22.4 \pm 2.1(18.3-28.1)$ & $25.9 \pm 3.4(18.9-39.0)$ & 0.021 \\
Normal weight, \% (n) & $88.4(38)$ & $38.2(26)$ & 0.018 \\
Overweight, \% (n) & $11.6(5)$ & $61.8(42)$ & \\
FSH $(\mathrm{mIU} / \mathrm{ml})$ & $6.5(5.0-11.2)$ & $6.1(3.2-10.3)$ & 0.019 \\
$\mathrm{LH}(\mathrm{mIU} / \mathrm{ml})$ & $6.85(3.85-9.45)$ & $9.78(5.63-19.33)$ & 0.046 \\
$\mathrm{PRL}(\mathrm{ng} / \mathrm{ml})$ & $11.96(6.89-22.13)$ & $37.39(8.93-66.56)$ & 0.031 \\
$\mathrm{E} 2(\mathrm{pg} / \mathrm{ml})$ & $32.9(28.1-45.8)$ & $77.1(52.4-93.6)$ & 0.029 \\
Glu $(\mathrm{mmol} / \mathrm{ml})$ & $4.8 \pm 1.2$ & $5.8 \pm 1.4$ & 0.011 \\
$\mathrm{~T}(\mathrm{ng} / \mathrm{ml})$ & $0.38 \pm 0.14$ & $0.84 \pm 0.21$ & 0.018 \\
Hyperandrogenemia, \% (n) & $8.8(6)$ & $57.3(39)$ & $0.023^{*}$ \\
Normal Androgen, \% (n) & $91.2(62)$ & $42.1(29)$ & \\
INS $(\mu \mathrm{H} / \mathrm{ml})$ & $7.8(4.6-21.7)$ & $22.9(8.4-29.3)$ & 0.047 \\
Hyperinsulinism, \% (n) & $4.4(3)$ & $45.6(31)$ & $0.042^{*}$ \\
Normal insulin, \% (n) & $95.6(65)$ & $54.4(37)$ & \\
\hline
\end{tabular}

Phase II: MiRNA validation

The validation of the 9 putative markers identified from the marker discovery phase was performed on a large set of serum samples from the left 68 pairs of pregnant women with PCOS and controls using RT-qPCR. Using cel-miR-39 as normalization control, our data demonstrated that three out of the 9 miRNAs (miR-222, miR-146a, miR-30c) in serum were significantly up-regulated in women with PCOS than healthy controls $(\mathrm{P}=0.014,0.024$, 0.031 for miR-222, miR-146a and miR-30c, respectively). The circulating levels of the other 6 miRNAs (miR-16, miR-106b, miR-19a, miR-24, miR-186 and miR-320) were not different between the two groups $(\mathrm{P}=0.058,0.074,0.069,0.067,0.086$ and 0.078 , respectively) (Table 3; Fig. 1).

Diagnostic value of maternal serum miRNAs for PCOS

To evaluate the sensitivity and specificity of the maternal serum miRNA signature individually and in combination for the prediction of PCOS, we further established ROC curves and calculated the AUC in each case (Fig. 2 A-C). We assessed the discriminating 
Table 3. Results of the serum miRNAs during the validation stage. *Mann-Whitney U test; \#The distribution

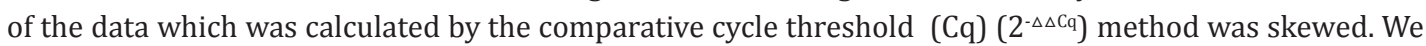
chose the median to represent the feature of the data. $\Delta \Delta \mathrm{Cq}=\Delta \mathrm{Cq}$ (case) $-\Delta \mathrm{Cq}$ control $\Delta \mathrm{Cq}=\mathrm{Cq}$ sample-Cq cel-miR-39

\begin{tabular}{|c|c|c|c|c|c|c|c|c|c|c|c|c|}
\hline \multirow[t]{2}{*}{ miRNA } & \multicolumn{5}{|c|}{ Control } & \multicolumn{5}{|c|}{ PCOS } & \multirow[t]{2}{*}{$\Delta \Delta \mathrm{Cq}$} & \multirow[t]{2}{*}{ P value* } \\
\hline & $\mathrm{N}$ & $\Delta \mathrm{Cq}$ & mean & SD & Median\# & $\mathrm{N}$ & $\Delta \mathrm{Cq}$ & mean & SD & median & & \\
\hline miR-222 & 43 & 5.89 & $1.68 * 10^{-2}$ & $1.32 * 10^{-2}$ & $1.21 * 10^{-2}$ & 68 & 3.57 & $8.42^{*} 10^{-2}$ & $4.61 * 10^{-2}$ & $5.86^{*} 10^{-2}$ & 2.42 & 0.014 \\
\hline miR-16 & 43 & 6.78 & $9.09 * 10^{-4}$ & $6.61 * 10^{-4}$ & $7.54 * 10^{-4}$ & 68 & 5.86 & $8.72^{*} 10^{-4}$ & $1.21 * 10^{-4}$ & $9.09 * 10^{-4}$ & 0.92 & 0.058 \\
\hline miR-19a & 43 & 4.96 & $3.21^{*} 10^{-3}$ & $2.25^{*} 10^{-3}$ & $2.67 * 10^{-3}$ & 68 & 4.42 & $4.67^{*} 10^{-3}$ & $1.19^{*} 10^{-3}$ & $2.88^{*} 10^{-3}$ & 0.54 & 0.074 \\
\hline miR-106b & 43 & 5.85 & $2.73^{*} 10^{-3}$ & $1.14^{*} 10^{-3}$ & $1.56^{*} 10^{-3}$ & 68 & 4.82 & $3.54^{*} 10^{-3}$ & $2.51^{*} 10^{-3}$ & $1.96 * 10^{-3}$ & 1.03 & 0.069 \\
\hline miR-30c & 43 & 6.63 & $1.01 * 10^{-2}$ & $7.85 * 10^{-3}$ & $9.2^{*} 10^{-3}$ & 68 & 5.16 & $2.79 * 10^{-2}$ & $1.72 * 10^{-2}$ & $2.69 * 10^{-2}$ & 1.47 & 0.024 \\
\hline miR-146a & 43 & 5.92 & $1.65^{*} 10^{-2}$ & $1.17 * 10^{-2}$ & $1.43 * 10^{-2}$ & 68 & 3.78 & $7.27^{*} 10^{-2}$ & $2.81^{*} 10^{-2}$ & $6.43 * 10^{-2}$ & 2.14 & 0.031 \\
\hline miR-24 & 43 & 7.89 & $4.22 * 10^{-4}$ & $2.25^{*} 10^{-4}$ & $3.98 * 10^{-4}$ & 68 & 6.96 & $5.03^{*} 10^{-4}$ & $2.94 * 10^{-4}$ & $4.81 * 10^{-4}$ & 0.93 & 0.067 \\
\hline miR-186 & 43 & 6.61 & $1.02^{*} 10^{-3}$ & $4.98^{*} 10^{-4}$ & $6.81 * 10^{-4}$ & 68 & 6.34 & $1.23^{*} 10^{-3}$ & $6.87 * 10^{-4}$ & $1.27^{*} 10^{-3}$ & 0.27 & 0.086 \\
\hline miR-320 & 43 & 6.24 & $1.32^{*} 10^{-2}$ & $1.16^{*} 10^{-2}$ & $7.24 * 10^{-3}$ & 68 & 7.31 & $8.37^{*} 10^{-3}$ & $5.71^{*} 10^{-3}$ & $8.68 * 10^{-3}$ & -1.07 & 0.078 \\
\hline
\end{tabular}

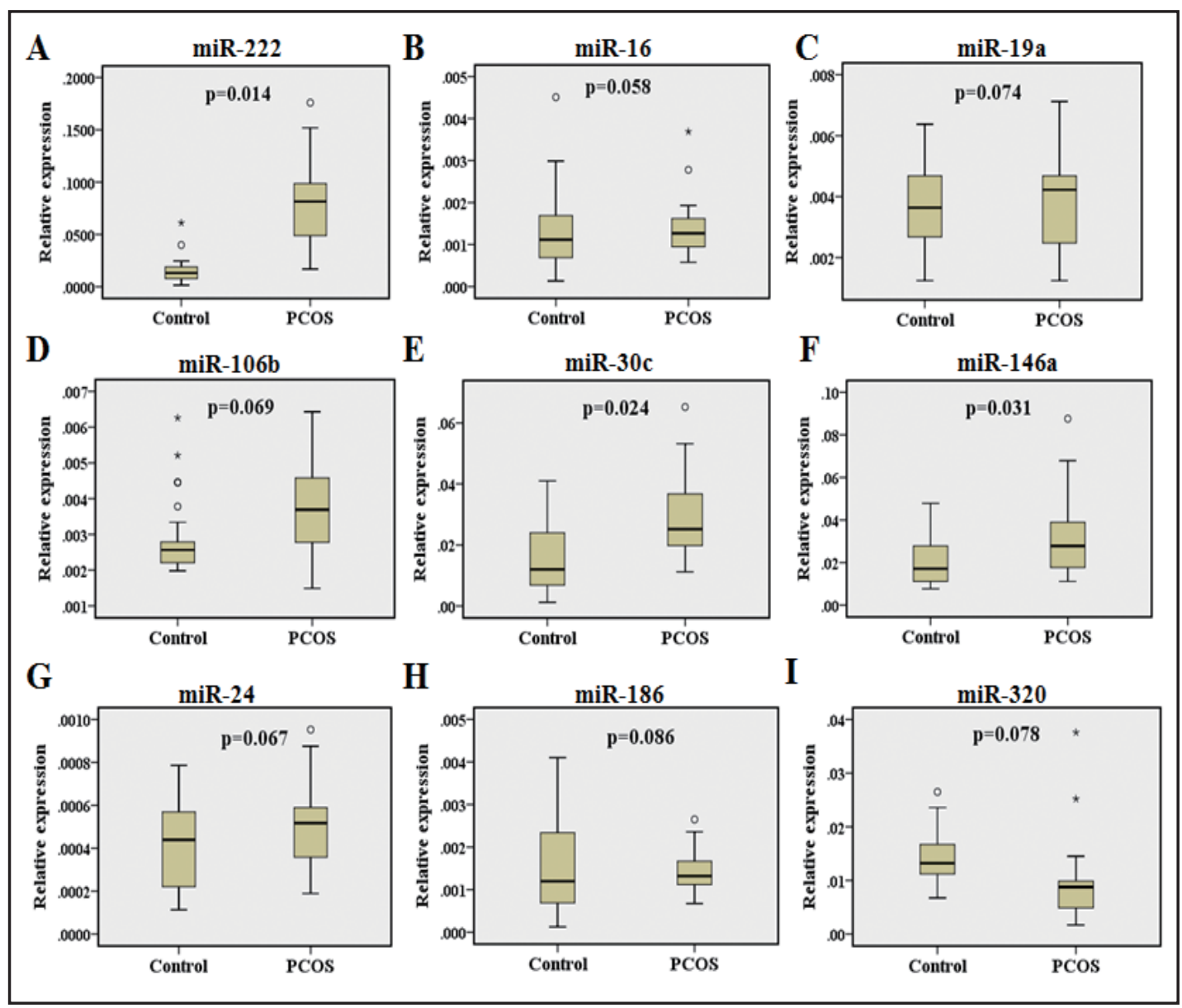

Fig. 1. Relative expression levels of 9 miRNAs in 68 pairs of women with PCOS and control subjects in the phase II. (A-D) Box plots of maternal serum miR-222, miR-16, miR-19a, miR-106b in 43 pairs of women with PCOS and healthy controls. (E-I) Box plots of the other 5 maternal serum miRNAs between the two groups. All the Box-whisker Plots represented the relative expression levels of miRNAs which were normalized against the spiked-in cel-miR-39 (A-K). The bottom and top of the box were the 5 th and $95^{\text {th }}$ percentiles, and the band in the middle of the box was the 50th percentile of the relative expression levels of miRNAs. Any data beyond these whiskers were shown as cycles and stars. 
$\mathbf{A}$

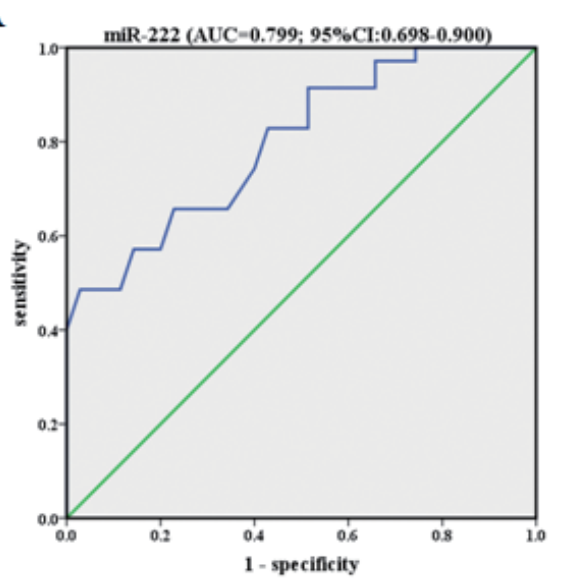

C

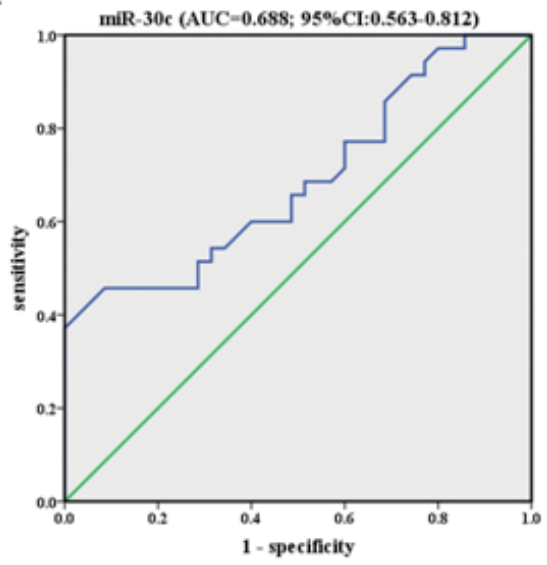

B

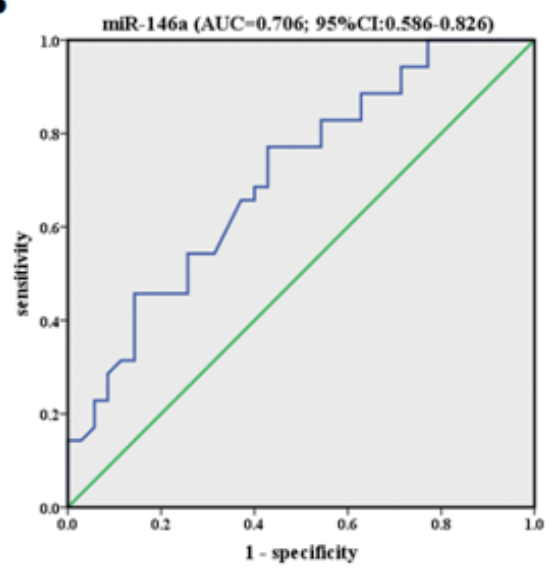

D

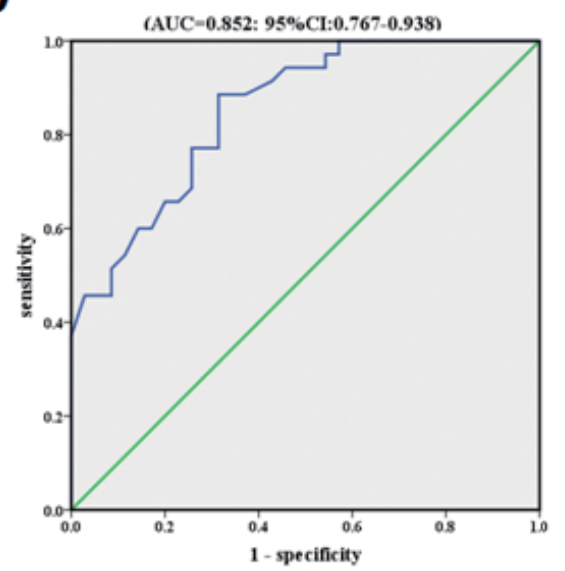

Fig. 2. ROC curve analysis using three miRNAs to discriminate women with PCOS from healthy controls. (A) miR-222, (B) miR-146a, (C) miR-30c, (D) ROC curve of the combination of the 3 miRNAs using multiple logistic regression analysis. The combination of the three miRNAs (miR-222, miR-146a and miR-30c) yielded the largest area under the ROC curve (AUC).

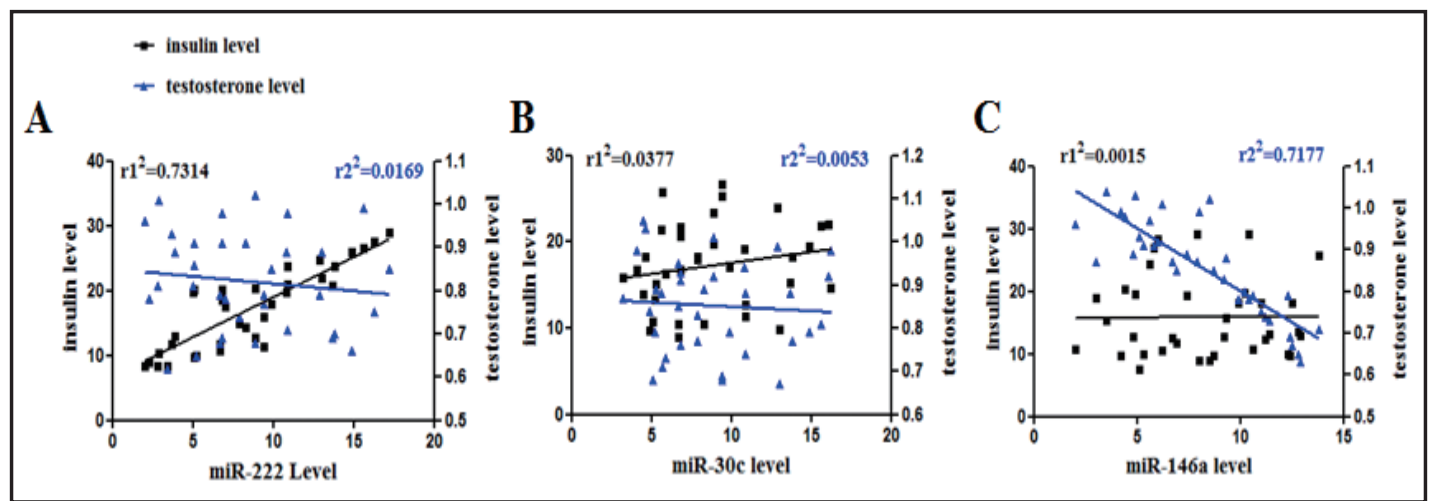

Fig. 3. Scatterplots of serum miRNA level vs insulin and testosterone level in PCOS women (A) miR-222, (B) miR-30c, (C) miR-146a.

effect of miR-222, miR-146a and miR-30c at the cut-off values of 1.28, 2.53 and 1.42, respectively, at which the largest Youden's index (sensitivity + specificity - 1) was defined as the optimal diagnostic point. As a consequence, the sensitivity and specificity of miR222, miR-146a and miR-30c were $73.2 \%$ and $75.6 \%, 62.9 \%$ and $84.6 \%, 71.5 \%$ and $64.2 \%$, 
Table 4. Gene ontology (GO) biological process and molecular function annotations associated with the gene targets of differentially expressed miRNAs $(\mathrm{P}<0.05)$. The top five GO biological processes and molecular functions are described for each individual miRNA including the number of gene targets represented in each classification

\begin{tabular}{|c|c|c|c|c|c|c|c|}
\hline MicroRNA & $\begin{array}{l}\text { No. of target } \\
\text { genes }\end{array}$ & GO biological processes & $\begin{array}{l}\text { No. of } \\
\text { genes }\end{array}$ & $P$ value & GO molecular function & $\begin{array}{l}\text { No. of } \\
\text { genes }\end{array}$ & $P$ value \\
\hline \multirow[t]{5}{*}{ hsa-miR-222 } & \multirow[t]{5}{*}{292} & protein complex biogenesis & 23 & $1.2 \mathrm{E}-4$ & kinase binding & 9 & $1.6 \mathrm{E}-2$ \\
\hline & & intracellular transport & 27 & $1.3 \mathrm{E}-4$ & transcription factor binding & 15 & $8.2 \mathrm{E}-2$ \\
\hline & & cell cycle & 30 & $1.5 \mathrm{E}-4$ & DNA binding & 52 & $7.9 \mathrm{E}-2$ \\
\hline & & posttranscriptional regulation of gene expression & 13 & $4.4 \mathrm{E}-4$ & aspartate-tRNA ligase activity & 5 & $7.0 \mathrm{E}-2$ \\
\hline & & negative regulation of RNA metabolic process & 16 & $2.3 \mathrm{E}-4$ & enzyme inhibitor activity & 11 & $2.6 \mathrm{E}-2$ \\
\hline \multirow[t]{5}{*}{ hsa-miR-146a } & \multirow[t]{5}{*}{97} & regulation of cytokine production & 10 & $5.9 \mathrm{E}-7$ & protein dimerization activity & 9 & $8.5 \mathrm{E}-3$ \\
\hline & & immune response & 19 & $2.2 \mathrm{E}-8$ & kinase binding & 7 & $1.6 \mathrm{E}-2$ \\
\hline & & regulation of programmed cell death & 21 & $2.5 \mathrm{E}-7$ & cytokine activity & 10 & $2.1 \mathrm{E}-2$ \\
\hline & & positive regulation of cell communication & 10 & $7.3 \mathrm{E}-5$ & lipopolysaccharide receptor activity & 4 & $2.7 \mathrm{E}-2$ \\
\hline & & positive regulation of cellular biosynthetic process & 14 & $7.7 \mathrm{E}-5$ & pattern recognition receptor activity & 6 & $7.3 \mathrm{E}-2$ \\
\hline \multirow[t]{5}{*}{ hsa-miR-30c } & \multirow[t]{5}{*}{213} & modification-dependent protein catabolic process & 22 & $2.6 \mathrm{E}-5$ & RNA binding & 23 & $2.6 \mathrm{E}-4$ \\
\hline & & ubiquitin-dependent protein catabolic process & 14 & $9.6 \mathrm{E}-5$ & protein kinase binding & 17 & $1.5 \mathrm{E}-2$ \\
\hline & & protein catabolic process & 23 & $8.1 \mathrm{E}-5$ & acid-amino acid ligase activity & 8 & $1.9 \mathrm{E}-2$ \\
\hline & & regulation of RNA metabolic process & 39 & $2.7 \mathrm{E}-3$ & structural constituent of ribosome & 7 & $2.6 \mathrm{E}-2$ \\
\hline & & negative regulation of gene expression & 16 & $3.0 \mathrm{E}-3$ & $2.6 \mathrm{E}-2$ & 11 & $5.3 \mathrm{E}-2$ \\
\hline
\end{tabular}

respectively (detailed data not shown). When we subjected the three miRNAs to multiple logistic regulation analysis, the AUC increased to 85.2\% (95\% CI: 0.767-0.938) (Fig. 2D). The results reflected that the combination of the three differentially expression miRNA which yielded the largest AUC showed more efficient as biomarker tool for the detection of PCOS.

The correlation of serum miRNA level and insulin and Testosterone

To evaluate independent associations between miRNA and androgens and glucose metabolites, we performed logistic binary regression analyses with adjustments for age and BMI. As a consequence, in PCOS women, miR-222 level were strongly and positively associated with serum insulin level $\left(\mathrm{r}^{2}=0.7314 ; \mathrm{P}<0.001\right)$, while the miR-146a was negatively associated with serum testosterone $\left(r^{2}=0.7177 ; \mathrm{P}<0.001\right)$. (Fig. 3)

\section{Functional classification of differentially expressed miRNA}

Enrichment of biological processes in terms of Gene Ontology categories with respect to the target genes was determined using the DAVID Bioinformatics Resources. The main GO biological processes and molecular function of the three miRNAs were summarized in Table 4.

In order to obtain a list of pathways likely to be specifically controlled by miRNAs significantly differentially expressed in the serum with PCOS and the controls, we performed an analysis of the most common signaling pathways by using the web-based computational tool named DIANA-miRPath. This computational tool estimates the impact of co-expressed miRNAs in biological pathways. As shown in Table 5, genes targeted by miR-222, miR-146a and miR-30 were found to be involved in metastasis, cell cycle, apoptosis and endocrine pathways, such as the Wnt signaling pathway, MAPK signaling pathway, Jak-STAT signaling pathway, etc.

\section{Discussion}

In the present study, for the first time, serum miRNA expression profiles of PCOS patients were analyzed and miRNAs that were specifically modulated in PCOS patients versus control subjects were identified. Additionally, ROC analysis indicated that miR-222, miR-146a and 
Table 5. The related pathways of predicted miRNA gene targets

\begin{tabular}{|c|c|c|c|c|}
\hline microRNA & KEGG pathways & Target gene & $\begin{array}{l}\text { Number } \\
\text { ofturgets }\end{array}$ & P value \\
\hline \multirow[t]{5}{*}{ hsa-mir-222 } & PI3K-Akt signaling pathway & $\begin{array}{l}\text { PPP2R5E, YWHAG, CREB1，PPP2R2A, } \\
\text { JAK3, PTEN, KDR, ITGA3 }\end{array}$ & 8 & 0.0015 \\
\hline & Pathways in cancer & $\begin{array}{l}\text { FOS, ARNT, ETS1, CDKN1B, AXIN2, KIT, } \\
\text { TFG }\end{array}$ & 7 & 0.0038 \\
\hline & VEGF signaling pathway & PPP3R1, NFATC3, KDR & 3 & 0.0037 \\
\hline & p53 signaling pathway & APAF1, BBC3, SESN3, PTEN & 4 & 0.010 \\
\hline & Wnt signaling pathway & $\begin{array}{l}\text { PPP2R5E, FRAT2, NLK, AXIN2, NFNTC, } \\
\text { WNT9A }\end{array}$ & 6 & 0.0045 \\
\hline \multirow[t]{5}{*}{ hsa-mir-146a } & Toll-like receptor signaling pathway & $\begin{array}{l}\text { TLR2, NFKB1, IL8, CD80, FADD, TRAF6, } \\
\text { CD86, IRF7, IRAK1 }\end{array}$ & 9 & $2.05 \mathrm{e}-13$ \\
\hline & Apoptosis & NFKB1, FADD, FAS, IRAK2, IRAK1, & 5 & $6.15 e-05$ \\
\hline & Cell adhesion molecules & CD80, CD86, CD40LG, ITGB2 & 4 & $4.62 \mathrm{e}-05$ \\
\hline & NF-kappa B signaling pathway & $\begin{array}{l}\text { NFKB1, IL8, CD40LG, TRAF6, BCL2A1, } \\
\text { IRAK1 }\end{array}$ & 6 & $2.61 \mathrm{e}-10$ \\
\hline & NOD-like receptor signaling pathway & NFKB1,IL8, TRAF6 & 3 & 0.010 \\
\hline \multirow[t]{5}{*}{ hsa-mir-30c } & MAPK signaling pathway & $\begin{array}{l}\text { MAPK8, RASA2, FGF20, KRAS, TAOK1, } \\
\text { CASP3, NFATC2, SOS1, MAP3K2, } \\
\text { PDGFA, ARRB1 }\end{array}$ & 11 & 0.0013 \\
\hline & Type II diabetes mellitus & $\begin{array}{l}\text { IRS2, SOCS3, PIK3CD, SOCS1, IRS1, } \\
\text { MAPK8 }\end{array}$ & 6 & 0.0003 \\
\hline & Cell cycle & $\begin{array}{l}\text { GSK3B, SMC1A, SMAD2, E2F2, CDC14A, } \\
\text { ORC2, MCM6, ORC4, DBF4, STAG2, } \\
\text { TFDP1, CCNE2 }\end{array}$ & 12 & $6.61 \mathrm{e}-05$ \\
\hline & PI3K-Akt signaling pathway & $\begin{array}{l}\text { GSK3B, FGF20, PAK1, FOX03, KDR, } \\
\text { PAK1, SOS1, EFNA3, IGF1R }\end{array}$ & 9 & 0.0089 \\
\hline & Wnt signaling pathway & $\begin{array}{l}\text { FZD7, GSK3B, MAPK8, NFAT5, SMAD2, } \\
\text { APC, DKK2, CAMK2D }\end{array}$ & 8 & 0.0011 \\
\hline
\end{tabular}

miR-30c may serve as novel biomarkers for diagnosis of PCOS. Furthermore, these data show a positive association between miR-222 and serum insulin, a negative association between miR-146a and serum testosterone.

PCOS is a syndrome of ovarian dysfunction. To date, the etiology of PCOS is still unclear, its clinical manifestations are also highly heterogeneous. Hyperandrogenism and polycystic ovary (PCO) morphology are known as cardinalfeatures [20]. Additionally, it may include: menstrual irregularities, signs of androgen excess, and obesity. Since the $1990 \mathrm{NIH}-$ sponsored conference on polycystic ovary syndrome (PCOS), it has become appreciated that the syndrome encompasses a broader spectrum of signs and symptoms of ovarian dysfunction than those defined by the original diagnostic criteria [21]. PCOS remains a syndrome and, as such, no single diagnostic criterion is sufficient for clinical diagnosis. The predictive power of endocrine variables in the diagnosis of PCOS has been assessed with receiver operating characteristic (ROC) analyses [22-25]. Despite inclusion of similar serum endocrine variables, this approach has yielded different diagnostic discriminators $[22,23$, 25].

Recently, several studies had reported that human serum/plasma could serve as a class of novel promising noninvasive biomarkers for diseases [12, 26-29]. Ng et al. found that the expression levels of plasma miR-92 were significantly elevated in colorectal cancer patients and well distinguished from gastric cancer, inflammatory bowel disease and normal subjects [27]. Plasma miR-1 expression level was found to be significantly higher in acute myocardial infarction (AMI) patients compared with non-AMI subjects, and the level was dropped to normal on discharge following medication [29]. 
For PCOS, a number of miRNAs have been reported to be differently expressed in the ovary tissues, such as miR-146a, miR-22, miR-132, miR-200c, miR-141, and miR-21 [30]. However, the serum miRNAs profile has not been reported. Our study identified 8 miRNAs up-regulated in the PCOS whereas 1 miRNAs had lower expression compared to controls in the discovery stage. Interestingly, most miRNAs that were differentially expressed in PCOS ovary tissues were not found modulated in serum of PCOS patient. Therefore, generally, the miRNAs in the PCOS ovary tissues were not all released into the blood. The exact mechanism how miRNAs enter into the serum and whether they are biologically functional or simply biomarkers for unknown etiological factors is still a big obsession. In this study, three miRNAs of miR-222, miR-146a and miR-30c were validated by RT-qPCR eventually. Among them, miR-222 has been shown a marked increase in Type 2 Diabetes and its potential relevance in insulin sensitivity [31]. Intriguingly, our study indicated that miR222 was positively associated with serum insulin. miR-146a was reported to suppress the release of progesterone, androgens, and estrogens. It was hypothesized that miR-146a act as physiological suppressors of general secretory activity [32]. In consistent with previous study, our study revealed that miR-146a was negatively associated with serum testosterone. Finally, bioinformatics analyses indicated genes targeted by miR-222, miR-146a and miR30 were found to be involved in metastasis, cell cycle, apoptosis and endocrine pathways, such as the Wnt signaling pathway, MAPK signaling pathway, Jak-STAT signaling pathway, etc. These data indicated that miR-222, miR-146a and miR-30 may be involved in the pathogenesis of PCOS.

Our study had several strengths. One of them is the community recruitment procedure, which allowed inclusion of PCOS women without previous health care contact, and rigorous inclusion/exclusion criteria. Secondly, we paid attention to the diagnosis of PCOS. It shed light on the objective, reliable and convenient effects compared to original diagnostic criteria. Thirdly, we used TaqMan Low Density Array, an easy and fast technology, to identify the differential miRNAs profile. As the gold standard way to quantify the expression of miRNAs, RT-qPCR only can detect the limited kinds of miRNAs one time, which probably makes it better as a validation rather than a discovery tool [33]. However, the TaqMan Low Density Array can now profile hundreds of miRNAs simultaneously using the highly optimized TaqMan chemistry. In addition, it provides several orders of magnitude greater sensitivity and a significantly broader dynamic range than microarray profiling. Lastly, we used celmiR-39 as a normalization strategy in phase II to replace the traditional tissue approach, which may have led to more accurate and reliable expression data regarding serum miRNAs. However, certain limitations still need to be addressed and should be mentioned. During phase I, we pooled the serum of five cases and five controls, respectively, to perform TaqMan Low Density Array. If these had been separated, a more typical microRNA panel for the validation stage might have been identified. Furthermore, the detailed mechanism and roles of these miRNAs remains unknown, and more fundamental studies are required.

In conclusion, we found that miRNA expression is altered in PCOS patients versus control subjects, suggesting a possible discrimination between healthy individuals and PCOS patients, by measuring a small number of miRNAs. Further studies are needed to assess whether the identified miRNAs may constitute a useful tool for patient diagnosis and treatment.

\section{Acknowlegement}

This work was financially supported by the major Foundation of Nanjing Medical University (2012NJMU184). It is also supported by the Medical Science and Technique Development Foundation of Nanjing Department of Health (YKK12099), the major science and technology of Nanjing Health Bureau(ZDX12009) and the Bureau of Nanjing City Science and Technology Development Found(201201063). 
Long et al.: Serum MicroRNAs Profile of PCOS

\section{Reference}

1 Dunaif A: Insulin resistance and the polycystic ovary syndrome: Mechanism and implications for pathogenesis. Endocr Rev 1997;18:774-800.

2 Norman RJ, Dewailly D, Legro RS, Hickey TE: Polycystic ovary syndrome. Lancet 2007;370:685-697.

- 3 Azziz R, Carmina E, Dewailly D, Diamanti-Kandarakis E, Escobar-Morreale HF, Futterweit W, Janssen OE, Legro RS, Norman RJ, Taylor AE, Witchel SF: The androgen excess and pcos society criteria for the polycystic ovary syndrome: The complete task force report. Fertil Steril 2009;91:456-488.

4 Revised 2003 consensus on diagnostic criteria and long-term health risks related to polycystic ovary syndrome (pcos). Hum Reprod 2004;19:41-47.

5 Franks S, McCarthy MI, Hardy K: Development of polycystic ovary syndrome: Involvement of genetic and environmental factors. Int J Androl 2006;29:278-285; discussion 286-290.

He L, Hannon GJ: Micrornas: Small rnas with a big role in gene regulation. Nat Rev Genet 2004;5:522-531. Li W, Ruan K: Microrna detection by microarray. Anal Bioanal Chem 2009;394:1117-1124. Pauley KM, Satoh M, Chan AL, Bubb MR, Reeves WH, Chan EK: Upregulated mir-146a expression in peripheral blood mononuclear cells from rheumatoid arthritis patients. Arthritis Res Ther 2008;10:R101. Jones SW, Watkins G, Le Good N, Roberts S, Murphy CL, Brockbank SM, Needham MR, Read SJ, Newham P: The identification of differentially expressed microrna in osteoarthritic tissue that modulate the production of tnf-alpha and mmp13. Osteoarthritis Cartilage 2009;17:464-472.

10 Teague EM, Print CG, Hull ML: The role of micrornas in endometriosis and associated reproductive conditions. Hum Reprod Update 2010;16:142-165.

-11 McCallie B, Schoolcraft WB, Katz-Jaffe MG: Aberration of blastocyst microrna expression is associated with human infertility. Fertil Steril 2010;93:2374-2382.

12 Chen X, Ba Y, Ma L, Cai X, Yin Y, Wang K, Guo J, Zhang Y, Chen J, Guo X, Li Q Li X, Wang W, Wang J, Jiang X, Xiang Y, Xu C, Zheng P, Zhang J, Li R, Zhang H, Shang X, Gong T, Ning G, Zen K, Zhang CY: Characterization of micrornas in serum: A novel class of biomarkers for diagnosis of cancer and other diseases. Cell Res 2008;18:997-1006.

13 Zhang Y, Liu D, Chen X, Li J, Li L, Bian Z, Sun F, Lu J, Yin Y, Cai X, Sun Q, Wang K, Ba Y, Wang Q, Wang D, Yang J, Liu P, Xu T, Yan Q, Zhang J, Zen K, Zhang CY: Secreted monocytic mir-150 enhances targeted endothelial cell migration. Mol Cell 2010;39:133-144.

14 Kroh EM, Parkin RK, Mitchell PS, Tewari M: Analysis of circulating microrna biomarkers in plasma and serum using quantitative reverse transcription-pcr (qrt-pcr). Methods 2010;50:298-301.

- 15 Mitchell PS, Parkin RK, Kroh EM, Fritz BR, Wyman SK, Pogosova-Agadjanyan EL, Peterson A, Noteboom J, O'Briant KC, Allen A, Lin DW, Urban N, Drescher CW, Knudsen BS, Stirewalt DL, Gentleman R, Vessella RL, Nelson PS, Martin DB, Tewari M: Circulating micrornas as stable blood-based markers for cancer detection. Proc Natl Acad Sci U S A 2008;105:10513-10518.

-16 Chen C, Ridzon DA, Broomer AJ, Zhou Z, Lee DH, Nguyen JT, Barbisin M, Xu NL, Mahuvakar VR, Andersen MR, Lao KQ Livak KJ, Guegler KJ: Real-time quantification of micrornas by stem-loop rt-pcr. Nucleic Acids Res 2005;33:e179.

17 Ashburner M, Ball CA, Blake JA, Botstein D, Butler H, Cherry JM, Davis AP, Dolinski K, Dwight SS, Eppig JT, Harris MA, Hill DP, Issel-Tarver L, Kasarskis A, Lewis S, Matese JC, Richardson JE, Ringwald M, Rubin GM, Sherlock G: Gene ontology: Tool for the unification of biology. The gene ontology consortium. Nat Genet 2000;25:25-29.

18 Papadopoulos GL, Alexiou P, Maragkakis M, Reczko M, Hatzigeorgiou AG: Diana-mirpath: Integrating human and mouse micrornas in pathways. Bioinformatics 2009;25:1991-1993.

19 Kanehisa M, Goto S: Kegg: Kyoto encyclopedia of genes and genomes. Nucleic Acids Res 2000;28:27-30.

20 Laven JS, Imani B, Eijkemans MJ, Fauser BC: New approach to polycystic ovary syndrome and other forms of anovulatory infertility. Obstet Gynecol Surv 2002;57:755-767.

21 Legro RS, Kunselman AR, Dunaif A: Prevalence and predictors of dyslipidemia in women with polycystic ovary syndrome. Am J Med 2001;111:607-613.

-22 Escobar-Morreale HF, Asuncion M, Calvo RM, Sancho J, San Millan JL: Receiver operating characteristic analysis of the performance of basal serum hormone profiles for the diagnosis of polycystic ovary syndrome in epidemiological studies. Eur J Endocrinol 2001;145:619-624. 
23 Koskinen P, Penttila TA, Anttila L, Erkkola R, Irjala K: Optimal use of hormone determinations in the biochemical diagnosis of the polycystic ovary syndrome. Fertil Steril 1996;65:517-522.

24 Turhan NO, Toppare MF, Seckin NC, Dilmen G: The predictive power of endocrine tests for the diagnosis of polycystic ovaries in women with oligoamenorrhea. Gynecol Obstet Invest 1999;48:183-186.

25 Pall ME, Lao MC, Patel SS, Lee ML, Ghods DE, Chandler DW, Friedman TC: Testosterone and bioavailable testosterone help to distinguish between mild cushing's syndrome and polycystic ovarian syndrome. Horm Metab Res 2008;40:813-818.

-26 Hu Z, Chen X, Zhao Y, Tian T, Jin G, Shu Y, Chen Y, Xu L, Zen K, Zhang C, Shen H: Serum microrna signatures identified in a genome-wide serum microrna expression profiling predict survival of non-small-cell lung cancer. J Clin Oncol 2010;28:1721-1726.

27 Ng EK, Chong WW, Jin H, Lam EK, Shin VY, Yu J, Poon TC, Ng SS, Sung JJ: Differential expression of micrornas in plasma of patients with colorectal cancer: A potential marker for colorectal cancer screening. Gut 2009;58:1375-1381.

28 Fichtlscherer S, De Rosa S, Fox H, Schwietz T, Fischer A, Liebetrau C, Weber M, Hamm CW, Roxe T, MullerArdogan M, Bonauer A, Zeiher AM, Dimmeler S: Circulating micrornas in patients with coronary artery disease. Circ Res 2010;107:677-684.

29 Ai J, Zhang R, Li Y, Pu J, Lu Y, Jiao J, Li K, Yu B, Li Z, Wang R, Wang L, Li Q, Wang N, Shan H, Yang B: Circulating microrna-1 as a potential novel biomarker for acute myocardial infarction. Biochem Biophys Res Commun 2010;391:73-77.

30 Baley J, Li J: Micrornas and ovarian function. J Ovarian Res 2012;5:8.

-31 Ortega FJ, Mercader JM, Moreno-Navarrete JM, Rovira O, Guerra E, Esteve E, Xifra G, Martinez C, Ricart W, Rieusset J, Rome S, Karczewska-Kupczewska M, Straczkowski M, Fernandez-Real JM: Profiling of circulating micrornas reveals common micrornas linked to type 2 diabetes that change with insulin sensitization. Diabetes Care 2014

-32 Sirotkin AV, Ovcharenko D, Grossmann R, Laukova M, Mlyncek M: Identification of micrornas controlling human ovarian cell steroidogenesis via a genome-scale screen. J Cell Physiol 2009;219:415-420.

-33 Git A, Dvinge H, Salmon-Divon M, Osborne M, Kutter C, Hadfield J, Bertone P, Caldas C: Systematic comparison of microarray profiling, real-time pcr, and next-generation sequencing technologies for measuring differential microrna expression. RNA 2010;16:991-1006. 